\title{
BMJ Open Effectiveness of an emergency nurse practitioner service for adults presenting to rural hospitals with chest pain: protocol for a multicentre, longitudinal nested cohort study
}

\author{
Tina E Roche, ${ }^{1,2}$ Glenn Gardner, ${ }^{2}$ Peter A Lewis ${ }^{2}$
}

To cite: Roche TE, Gardner G, Lewis PA. Effectiveness of an emergency nurse practitioner service for adults presenting to rural hospitals with chest pain: protocol for a multicentre, longitudinal nested cohort study. BMJ Open 2015;5:e006997. doi:10.1136/bmjopen-2014006997

- Prepublication history and additional material is available. To view please visit the journal (http://dx.doi.org/ 10.1136/bmjopen-2014006997)

Received 23 October 2014 Revised 8 January 2015 Accepted 12 January 2015

\section{CrossMark}

${ }^{1}$ Stanthorpe Health Services, Emergency Department, Stanthorpe, Queensland, Australia

${ }^{2}$ School of Nursing, Queensland University of Technology, Institute of Health and Biomedical Innovation, Kelvin Grove, Queensland, Australia

Correspondence to

Tina E Roche;

tina.roche@hdr.qut.edu.au

\section{ABSTRACT}

Introduction: Chest pain is common in emergency department (ED) patients and represents a considerable burden for rural health services. Health services reforms to improve access to care need appropriately skilled and supported clinicians in the delivery of safe and effective care, including the use of emergency nurse practitioners (ENPS). Despite increasing use of ENPs, little is known about the safety and quality of the service in the rural ED context. The aims of this study are (1) to examine the safety and quality of the ENP service model in the provision of care in the rural environment and (2) to evaluate the effectiveness of the service in the management of patients presenting with undifferentiated chest pain. Methods and analysis: This is the protocol for a prospective longitudinal nested cohort study to compare the effectiveness of ENP service with that of standard care. Adults presenting to three rural EDs in Queensland, Australia with a primary presenting complaint of atraumatic chest pain will be eligible for enrolment. We will measure (1) clinician's use of evidence-based guidelines (2) diagnostic accuracy of ECG interpretation for the management of patients with suspected or confirmed ACS (3) service indicators of waiting times, length-of-stay and did-not-wait rates and (4) clinician's diagnostic accuracy as measured by rates of unplanned representation within 7 days (5) satisfaction with care, (6) quality-of-life and (7) functional status. To assess these outcomes we will use a combination of measures collected from routinely collected data, medical record review and questionnaires (with 30-day follow-up).

Ethics and dissemination: Queensland Health Human Research Ethics Committee (HREC) has approved this protocol. The results will be published in peer-reviewed scientific journals and presented at one or more scientific conferences.

\section{INTRODUCTION}

People living in rural areas have shorter lives and poorer health outcomes when compared

\section{Strengths and limitations of this study}

- Beyond the context of minor injury and illness presentations in the metropolitan setting, the safety and quality of emergency nurse practitioner (ENP) service is not well researched and is poorly understood. This study is the first to evaluate rural ENP service in the management of a higher acuity, time sensitive presentation like chest pain.

- A prospective longitudinal nested cohort design was chosen to evaluate real world effectiveness in the context of a safety and quality framework using strategies to eliminate bias that is common to this methodology.

- A potential limitation of the study is the small ENP sample size that may affect the generalisability and external validity of the results of this study.

to people living in major cities, are more likely to be overweight, lead sedentary lifestyles and engage in risky behaviours like smoking and drinking alcohol in harmful quantities. ${ }^{1}$ It is likely that a combination of inequity in access to health services, risk factors and the rural environment are responsible for poorer rural health outcomes. ${ }^{1}$

Chest pain represents 5-10\% of Australian annual emergency department (ED) presentations $^{23}$ and is responsible for a quarter of all hospital admissions. ${ }^{3}$ Chest pain is symptomatic of many presenting aetiologies, one of which is acute coronary syndrome (ACS). This classification encompasses a broad spectrum of clinical presentations that includes acute myocardial infarction through to a pattern of angina without evidence of damage to the heart muscle. ${ }^{4}$ Given that acute myocardial infarction is the leading cause of sudden death in the Australian population, ${ }^{5}$ undifferentiated chest pain is a presentation of significance in EDs. 
While chest pain is a characteristic of ACS, the majority of patients with chest pain are ultimately found to have non-cardiac diagnoses. ${ }^{2}{ }^{6-8}$ Not-with-standing the diagnostic outcome, there is considerable cost to health services in evaluating patients who are experiencing undifferentiated chest pain. The challenge for clinicians and health services in caring for this patient cohort is to provide assessment and management with a high degree of safety in a timely and cost-effective manner in an era of increasing service demand. ${ }^{9}$ Strategies to reduce delays to testing, selection of patients for outpatient evaluation and assessment protocols that expedite evaluation and early specialist review are necessary. ${ }^{7}$

The rural context of care impacts on the capacity of health services to deliver care to patients presenting to EDs with chest pain. There are lower numbers of healthcare professionals in rural areas and most hospitals do not employ dedicated staff within the ED. Health service usage differs between major cities and rural locations due in part to the lower rates of general practitioner consultations and higher rates of hospital admissions. ${ }^{1}$ This has resulted in a call for rural health service reform to improve access by using an appropriately skilled and supported workforce in the delivery of quality care that is effective, appropriate and sustainable. ${ }^{10}$

There are many examples of innovative health service models being implemented throughout Australia including the use of expanded roles in nursing with the introduction of nurse practitioners (NPs). NPs have specialist skills and practice in an advanced nursing role with legislated extensions to practice. The emergency nurse practitioner (ENP) service model is the fastest growing NP specialty group in Australia with $61 \%$ growth in numbers over a 3-year period. ${ }^{11}$ In rural Australian EDs, there is growing use of this service with $38 \%$ of these departments now staffed by ENPs. ${ }^{12}$ While the ENP model has been utilised in rural areas to meet the need for accessible, quality care, little is known about the safety and quality of the service in this context.

To date there is no indication of published research investigating the effectiveness of ENP service in the management of patients presenting with complex medical needs in the rural context. This planned study, "Managing Chest Pain in Rural Emergency Departments", will address the gap in research by providing knowledge on ENP service and the processes and outcomes of care for rural patients experiencing undifferentiated chest pain.

\section{METHODS AND ANALYSIS}

Study aims

The aim of this study is to examine the safety and quality of the ENP service model in provision of care in the rural environment and to evaluate the effectiveness of the service in the management of patients presenting with undifferentiated chest pain. We plan to investigate several outcomes in order to address the following research questions:
1. What are the health service structures that influence the ENP delivery of safe, quality care for patients presenting to rural EDs with chest pain?

2. Are the processes of care for patients who present to rural EDs with chest pain equivalent for patients managed by an ENP service to those managed in the standard model of care?

3. Are the comparative outcomes for patients who present to rural EDs with chest pain equivalent for patients managed by an ENP service to those managed in the standard model of care?

\section{Study conceptual framework}

Evaluation of the safety and quality of the use of ENPs as a service innovation calls for an approach that can accommodate the complexity of multiple dimensions of a service improvement initiative. ${ }^{13}$ The Donabedian Framework $^{14}$ provides a model that supports systematic evaluation of healthcare services and will be used to guide data collection and inform interpretation of the study findings. According to Donabedian, there are three dimensions from which conclusions about the quality of care can be drawn; these are structure, process and outcome. ${ }^{14}$ Structure refers to the attributes of the healthcare setting (material, human and organisational resources), Process refers to what is actually done in the giving and receiving of healthcare and Outcome refers to the effects of healthcare on patients and populations ${ }^{15}$ (see figure 1). In essence the model asserts that quality in healthcare is possible because there is a relational effect; good structures increase the likelihood of good processes, which increases the likelihood of good outcomes. The framework is one of the best-known and widely used conceptual models for health services research ${ }^{16}$ and provides a basis for a rigorous, multidimensional evaluation of this service innovation. Examination of the structure of care for patients with chest pain who are managed by the ENP service is required to identify the limitations and advantages of this model of care. Evaluating the process and outcomes of care for the ENP service for this cohort of patients will assist in determining the quality of care provided. Further, by using a safety and quality

\section{Structure}

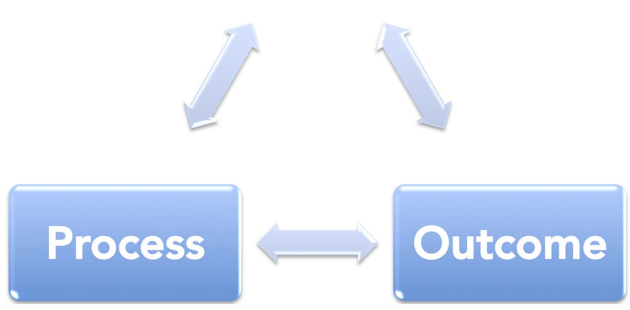

Figure 1 Donabedian's structure-process-outcome framework. 
framework, the strengths and weaknesses of each of the dimensions and the implications for the safety and quality of the service may be identified.

\section{Design}

This project is a prospective multicentre longitudinal cohort study. The study cohort is defined as adult patients presenting with atraumatic chest pain. A cohort design was chosen for this study to allow for an evaluation of the service model that is inclusive of multiple dimensions including the structure, process and outcome of the service and its context. Although randomised controlled trials (RCTs) are considered the 'gold standard' for research, in the area of healthcare, they cannot always be easily conducted. ${ }^{17}{ }^{18}$ RCTs are not always suited to evaluating complex combinations of service interventions and practices in terms of their real world effectiveness ${ }^{19}{ }^{20}$ in the context of a safety and quality framework. By using a cohort design, a specific population (rather than an isolated intervention or treatment) can be studied using multiple outcomes related to one or more exposures. ${ }^{17} 20$ Additionally, to address the research questions, a nested cohort will be identified from the study cohort and will consist of patients with International Classification of Diseases discharge diagnoses codes I20-I25 from the group ischaemic heart diseases. ${ }^{21}$ Specific diagnoses may include, but are not limited to, possible cardiac chest pain, angina pectoris, ACS and myocardial infarction. Using this nested cohort allows for statistically efficient analysis of data with substantial time savings. ${ }^{17}$

\section{Independent variable}

The independent variable is the service model involved in the management of patients presenting with undifferentiated chest pain. For the purpose of this study the models are operationally defined as follows:

- Emergency nurse practitioner model: The ENP manages the patient presenting with undifferentiated chest pain. The ENP delivers and coordinated care in the diagnosis, investigation, therapeutic treatment (including prescribing of medications and technical interventions) and referral. In this model ED nursing staff work with the ENP in providing nursing care to the patient.

- Standard care model: In this traditional model, all care for the patient presenting with undifferentiated chest pain is delivered and coordinated by a medical officer. In this model ED nursing staff work with the medical officer in providing nursing care to the patient.

In both models all clinicians work collaboratively and within their designated scope of practice.

\section{Outcome variables}

Outcome measures take into account the Donabedian SPO Framework. To assess the effectiveness of ENP service in the management of patients presenting to rural EDs with undifferentiated chest pain, we will measure and compare with standard care the following outcomes:
1. Use of evidence-based guidelines for the management of patients within the (Nested cohort) (Primary outcome variable)

2. Diagnostic accuracy of ECG interpretation (Nested cohort)

3. Service indicators of waiting times, length-of-stay and did-not-wait rates (Study cohort)

4. Diagnostic accuracy as measured by rates of unplanned representation within 7 days (Study cohort)

5. Satisfaction with care (Study cohort)

6. Quality-of-life (Study cohort); and

7. Functional status (Study cohort).

The extraneous variable for this study is ENP service; outcomes will assess the structural characteristics of the model, including:

1. Barriers and facilitators for ENP practice

2. Professional characteristics (years of experience)

3. Psychosocial characteristics (perceived role competence)

\section{Setting}

The study will take place in three rural hospital EDs, of differing size, in Queensland, Australia. There are approximately 26000 ED presentations yearly for Hospital A, 21000 for Hospital B and 8000 for Hospital C. These EDs have similar service capabilities including staff mix, available health technologies and referral strategies. Both onsite doctors and ENPs staff each facility. There are varying levels of experience in the medical and ENP staff that includes newly qualified staff through to veteran clinicians. Furthermore, all sites have ENP service and standard medical care for the management of patients presenting with undifferentiated chest pain. There are no specialist cardiac services at any of these EDs and each facility is located more than $150 \mathrm{~km}$ from the closest cardiac interventional hospital. Collaborative arrangements with specialist medical services for consultation and acute interhospital transfer are similar for medical and ENP service at each facility.

As this research is an observational study, there will be no allocation of intervention; rather the care delivery model will follow the standard method of patient allocation. The current practice at each of these facilities involves the use of the Australasian Triage Scale to ensure that patients are treated in order of clinical urgency. The next available clinician (ENP or medical officer) is responsible for providing care to patients in order of clinical urgency. Medical and ENP service is provided in and out of hours.

\section{Participants}

\section{Inclusion criteria}

Patients who present to the participating hospital EDs with chest pain during the data collection period will be eligible for recruitment, if they:

1. Are at least 18 years old;

2. Have chest pain that is not the result of an acute injury; 
3. Are capable (or have a legally acceptable representative) of providing informed consent.

\section{Participant recruitment}

There are two participant groups in the study; ENPs and patients.

ENP recruitment will be conducted at the start of the study. ENPs from each participating site will be invited to participate and supplied with study information and consent documents. On providing informed consent, ENPs will be requested to complete a self-administered questionnaire.

Patient recruitment will start in November 2014 and will continue through until May 2015. At the index presentation, presenting patients who meet the inclusion criteria will be identified by the triage nurse or treating clinician and invited to participate in the study. Participation in this research will involve the completion of a patient questionnaire at baseline, the researcher's use of routinely collected data and completion of follow-up patient questionnaire. Potential participants will receive information and consent package, explaining the purpose of the research and procedures involved in completing the study. Trained research assistants will explain the study, enrol eligible consenting patients and assist with the completion of a baseline questionnaire. Patients will be advised that they may decline to engage in the study or withdraw from participation at any time without disadvantage.

Data will be collected at the ED where patients are seeking care for their acute chest pain. While it is envisaged the majority of patients will be able to provide consent, some may be critically unwell and initially lack capacity to provide informed consent because of the emergent nature of their illness. Where a lack of capacity is deemed to be temporary and is expected to resolve in the course of treatment, consent will be sought from a legally acceptable representative (including the patient's relatives). When the patient recovers capacity, the patient will confirm consent (or not) as soon as practicable after the initial emergency has passed. If once the patient has regained capacity he/she withholds consent then that patient and their data will be withdrawn from the study.

\section{Data collection}

After informed consent is obtained, baseline data regarding demographic and clinical information will be collected for the study cohort. Minimal demographic data will be collected on patients who decline to participate to allow comparison to evaluate the homogeneity of the study sample. Baseline data will be used for two purposes. First, demographic data will provide information that will be used to control for potential confounders in statistical analysis. Second, using the diagnosis assigned by the treating clinician as determined at the time of discharge from the ED, patients will be identified for inclusion in the nested cohort. Data for the nested cohort will be collected from the medical record.
At the completion of the occasion-of-service, all study participants will be requested to complete a selfadministered questionnaire that will measure patientreported outcomes including satisfaction, quality-of-life and functional status. Data for unplanned representations to the ED will be collected 7 days after the index presentation.

Follow-up questionnaires will be posted to all study participants at 30 days after the index ED presentation.

The flow diagram for patient recruitment and data collection during the study is provided in figure 2 .

\section{Instruments}

This research will use a variety of methods to assess study outcomes including the use of routinely collected demographic and clinical data, medical record review and questionnaires (see table 1). To ensure reliable and unbiased extraction of data from the medical record review, research assistants will be trained in the use of data abstraction tools that have been designed for this study.

ENP questionnaire: A self-administered questionnaire of participating ENPs will be used to evaluate the structural characteristics of the service that are perceived barriers or enablers to practice. The questionnaire uses a component of the National Nurse Practitioner Survey that was developed in the Australian Nurse Practitioner Study (AusPrac). ${ }^{22}$

Baseline patient-reported outcomes questionnaire: This study will use an adaptation of the patient outcomes tools that were developed and/or incorporated from published work for the AusPrac Study. ${ }^{22}$ With permission of the authors, this study will adapt the AusPrac patient outcomes scales to assess patient satisfaction, experience with coordination of care, quality of life and functional status. Functional health and well-being will be measured using the SF-12, a copyrighted instrument of QualityMetric Incorporated. ${ }^{23}$ Internationally, the SF-12 survey has demonstrated reliability and validity, ${ }^{24}{ }^{25}$ including in Australia. ${ }^{26} 27$ The instrument has been using previously for investigation of patients with non-cardiac chest pain ${ }^{28}$ and for patients managed by the ENP service in Australia. ${ }^{22}{ }^{29}$ Permission to use this instrument for the study has been provided by the copyright holder.

Follow-up patient-reported outcomes questionnaire: These questionnaires will assess patient-reported outcomes using the modified AusPrac patient outcome scales and the SF-12.

Data abstraction tool for study cohort: A tool that utilises routinely collected data has been developed for the study (see online supplementary file 1). Data collected includes Australasian Triage Score, treating clinician category, diagnosis at discharge and discharge destination, service indicators including waiting time, length-of-stay, did-not-wait and unplanned representations will also be collected.

Data abstraction tool for nested cohort: Data will be collected from the participant's medical record using a tool that has been designed for the study (see online supplementary file 2). The tool uses criteria from the Clinical 


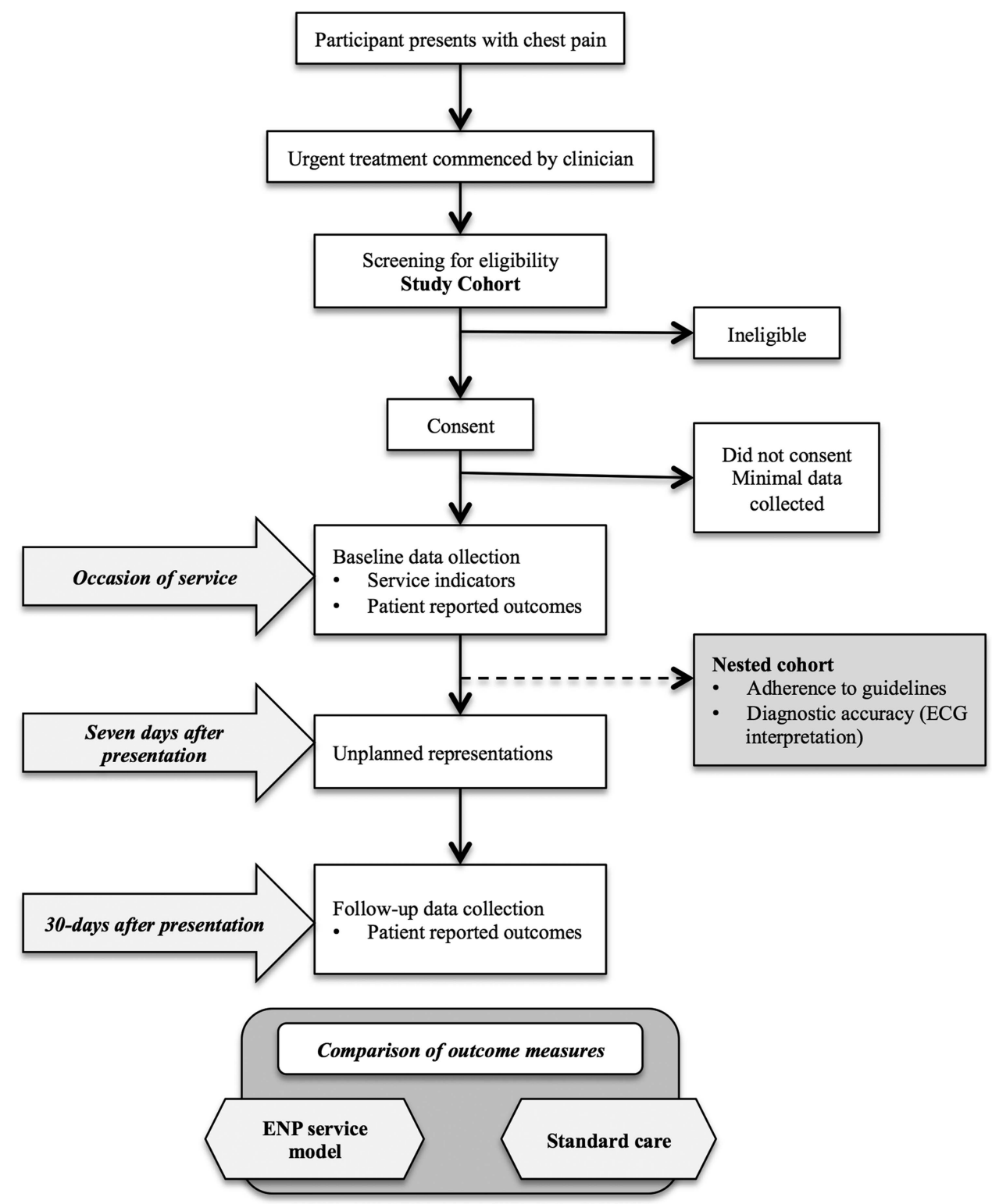

Figure 2 Flow diagram for patient recruitment and data collection. ENP, emergency nurse practitioners.

Pathway currently in use in Queensland Health facilities. These clinical pathways are used in all participating study sites and are based on the best practice recommendations of the National Heart Foundation/Cardiac Society of Australia and New Zealand Guidelines for suspected or confirmed acute coronary syndromes. ${ }^{4}$ Data will be collected to evaluate clinician's adherence to evidence-based guidelines, including pharmacological management, risk stratification and referral strategies. Where data is missing from the medical record (eg, evidence of administration of aspirin is not recorded) the intervention will be assumed not to have occurred. For the purposes of this study, cardiac biomarker testing that occurs at any time during the ED stay will be assessed as being 'on arrival' and in accordance with current guidelines. A copy of the participant's ECG/s will be collected. A blinded assessor who has specialist qualifications in emergency medicine will examine the treating clinician's interpretation of the diagnostic ECG for diagnostic accuracy.

\section{Sample size calculation}

There are an estimated 4730 total ED presentations across all participating sites each month. According to findings from a previous study, ${ }^{30}$ undifferentiated chest pain made up $3.5 \%$ of these and $39 \%$ of this group were cardiac related. Using these findings, there are approximately 65 patients with cardiac-related chest pain presenting to each of the participating EDs per month. Therefore in order to achieve the requisite sample, recruitment will be conducted over a 6 -month period.

The sample size calculations were based on $80 \%$ power and a type I error rate (two sided) of 0.05 . Sample size estimation was calculated for the nested cohort that will be used to evaluate the primary outcome of use of evidence based guidelines for patients 


\begin{tabular}{|c|c|c|}
\hline $\begin{array}{l}\text { Time } \\
\text { period* }^{*}\end{array}$ & Data source & Data collected \\
\hline $\begin{array}{l}\text { Start of } \\
\text { study }\end{array}$ & ENP questionnaire & $\begin{array}{l}\text { Structural } \\
\text { characteristics of the } \\
\text { service }\end{array}$ \\
\hline \multirow[t]{3}{*}{ Baseline } & $\begin{array}{l}\text { Patient } \\
\text { questionnaire }\end{array}$ & $\begin{array}{l}\text { Demographic data } \\
\text { Patient-reported } \\
\text { outcomes }\end{array}$ \\
\hline & Medical record & Clinical data \\
\hline & $\begin{array}{l}\text { Routinely-collected } \\
\text { data }\end{array}$ & Service indicators \\
\hline 7 days & $\begin{array}{l}\text { Routinely-collected } \\
\text { data }\end{array}$ & $\begin{array}{l}\text { Unplanned } \\
\text { representations }\end{array}$ \\
\hline 30 days & $\begin{array}{l}\text { Patient } \\
\text { questionnaire }\end{array}$ & $\begin{array}{l}\text { Patient-reported } \\
\text { outcomes }\end{array}$ \\
\hline
\end{tabular}

with cardiac-related chest pain. This calculation was based on (1) perusal of prior research studies together with unpublished local data to determine the rate of protocol compliance expected in cardiac chest pain patients at an estimated $50 \%$, (2) the proportion of cardiac chest pain patients who were seen by ENPs was identified as $25 \%$ and (3) the difference in protocol compliance between ENPs and doctors is expected to be larger than 20\%. The sample size calculated for the primary outcome cohort study under these assumptions is 384 patients with cardiac-related chest pain with an OR of 2.25 .

\section{Data analysis}

The conventional $5 \%$ level of statistical significance will be used. All analyses will be conducted using de-identified patient data using SPSS software (IBM SPSS Statistics) V.22.

- Structural characteristics of the ENP service model: Descriptive statistics will be used to summarise the data for structural characteristics of the ENP service model. Categorical data will be displayed as a proportion for each of the components of the survey.

- Patient Demographic and clinical data: Baseline characteristics potentially associated with study outcomes (age, gender, education level, employment, ATSI status, previous health service usage) will be reported separately for each service model. The data collected will be analysed using descriptive statistics. Dichotomous and nominal data will be displayed as a proportion; comparison of clinical data will be examined and tested for significance using the $\chi^{2}$ test.

- Service indicators and unplanned representation within 7 days: Descriptive statistics will be used. Continuous data will be used for analysis of waiting times and length-of-stay. Normally distributed data will report means and SDs; comparisons between service models will be examined using the unpaired t test. Data not normally distributed will be analysed using medians and IQR; comparisons between the two models will be tested for statistical significance using the MannWhitney test. The dichotomous data for unplanned representations will be displayed as an OR; comparison between the service models will be examined and tested for significance using the $\chi^{2}$ test.

- Adherence to evidence-based guidelines: Descriptive statistics will be used to summarise the adherence to guidelines for patients with suspected or confirmed ACS. A blinded assessor who has specialist qualifications in emergency medicine will undertake independent interpretation of ECGs, which will be compared to the clinician's interpretation. Dichotomous data will be displayed as a percentage of agreement proportion; comparisons between the service models will be examined and tested for significance using McNemar's test.

- Patient-reported outcomes: Data will be summarised and measures of distribution for patient-reported health outcomes will be conducted. Nominal and ordinal data collected for analysis of patient satisfaction will be displayed as a proportion; comparisons between the two service models will be examined and tested for significance using the $\chi^{2}$ test. The data for the SF-12 summary scores will be managed and analysed according to the guidelines from the SF tools and will be reported using means and SDs (for normally distributed data) or medians and IQR (for not normally distributed data). Comparisons between the service models will be tested for statistical significance. Regression analyses will evaluate the associations between functional status and other influencing factors.

\section{ETHICS AND DISSEMINATION}

Standard procedures for the protection of confidential individual information will be followed in accordance with national and international ethical recommendations and guidelines as well as relevant legislation.

The results of this study will provide evidence of the safety and quality of the ENP service model. The findings will be disseminated locally to inform health service planning and future recommendations for practice. Manuscripts arising from the study results will be submitted to peer-reviewed scientific journals and conference presentations will be prepared for Australian and international conferences.

\section{DISCUSSION}

Studies supporting the use of ENP service are mostly conducted in the context of minor injury and illness presentations and in metropolitan settings. Beyond this context, the safety and quality of ENP service is not well researched and is poorly understood. We have described the protocol for a longitudinal nested cohort study, The Managing Chest Pain in Rural Emergency Departments, which will examine the effectiveness of ENP service in the management of patients presenting to rural EDs 
with undifferentiated chest pain. This study is one of the first to evaluate rural ENP service in the management of a higher acuity, time sensitive presentation like chest pain.

Although RCTs are considered the 'gold standard' for research, a cohort design was chosen for this study because the guiding framework necessitates an evaluation of the service model that is inclusive of multiple dimensions that could not easily or ethically conducted in this study setting. Selection bias will be minimised by the use of a clearly defined study population and inclusion criteria. The study has been designed to avoid losses to follow-up and is conducted over a relatively short period of time. Information bias has been avoided by the use of clear, specific, measurable outcomes that will be accurately and consistently measured. The study will combine detailed information from routinely collected data, participants' medical record and questionnaire with repeated follow-up measurement from patients presenting to rural EDs with chest pain. Questionnaires have been developed using validated scales and tools.

Examination of the clinical care provided for this cohort of patients will contribute to the understanding of processes and outcomes for patients presenting to rural hospitals with undifferentiated chest pain. Using a longitudinal approach, the study will provide knowledge on the management of patients presenting to rural EDs with chest pain and the effectiveness of ENP service in the rural context.

A potential limitation of the study is that although the study is powered to demonstrate statistically significant differences between service models, the ENP sample size is small and may affect the generalisability and external validity of the results of this study.

In conclusion, while the timely delivery of quality patient care in the ED has emerged as one of the most important service indicators to be measured in contemporary healthcare, there are significant gaps in the research evaluating ENP service on the outcomes and processes of care for patients. Despite the increasing use of ENPs in rural areas, there is scant research reported in the national and international literature regarding ENPs in the rural ED. There is also a scarcity of research that has evaluated the model outside of the minor injury and illness context. The management of patients presenting to rural EDs with chest pain is under researched and poorly reported the literature. This research will provide a new information specific to this service and will assist in providing an evidence base for this innovation at a level that has not been studied before.

\section{Twitter Follow Tina Roche at @EDNursePractitioner@Y7Tina}

Contributors TER conceived and designed the study with GG and PAL. TER wrote the manuscript, and GG and PAL revised it critically for important intellectual content. All authors approved the final manuscript.

Competing interests None.

Ethics approval Ethical approval for the study has been granted by Queensland Health Human Research Ethics Committee (reference:
HREC/14/QHC/30) and the Queensland University of Technology Human Research Ethics Committee (reference: 1400000709).

Provenance and peer review Not commissioned; externally peer reviewed.

Data sharing statement This manuscript describes the study protocol for research that is yet to begin.

Open Access This is an Open Access article distributed in accordance with the Creative Commons Attribution Non Commercial (CC BY-NC 4.0) license, which permits others to distribute, remix, adapt, build upon this work noncommercially, and license their derivative works on different terms, provided the original work is properly cited and the use is non-commercial. See: http:// creativecommons.org/licenses/by-nc/4.0/

\section{REFERENCES}

1. Australian Institute of Health and Welfare. Health sector national minimum datasets. Retrieved from 2010. http://meteor.aihw.gov.au/ content/index.phtml/itemld/344850

2. George T, Ashover S, Cullen L, et al. Introduction of an accelerated diagnostic protocol in the assessment of emergency department patients with possible acute coronary syndrome: the Nambour Short Low- Intermediate Chest pain project. Emerg Med Australas 2013;25:340-4.

3. Than M, Parsonage WA, Frampton C, et al. Development and validation of the Emergency Department Assessment of Chest pain Score and $2 \mathrm{~h}$ accelerated diagnostic protocol. Emerg Med Australas 2014;26:34-44.

4. Chew DP, Ruta L-AM, Aroney CN, et al. 2011 Addendum to the National Heart Foundation of Australia/Cardiac Society of Australia and New Zealand Guidelines for the management of acute coronary syndromes (ACS) 2006. Heart Lung Circ 2011;20:487-502.

5. Kinsman LD, Rotter T, Willis J, et al. Do clinical pathways enhance access to evidence-based acute myocardial infarction treatment in rural emergency departments? Aust $J$ Rural Health 2012;20:59-66.

6. Cullen L, Mueller C, Parsonage WA, et al. Validation of highsensitivity troponin I in a 2-hour diagnostic strategy to assess 30-day outcomes in emergency department patients with possible acute coronary syndrome. J Am Coll Cardiol 2013;62:1242-9.

7. Groarke J, O'Brien J, Go G, et al. Cost burden of non-specific chest pain admissions. Ir J Med Sci 2013;182:57-61.

8. Meek R, Braitberg G, Nicolas C, et al. Effect on emergency department efficiency of an accelerated diagnostic pathway for the evaluation of chest pain. Emerg Med Australas 2012;24:285-93.

9. Parsonage WA, Cullen L, Younger JF. The approach to patients with possible cardiac chest pain. Med J Aust 2013;199:30.

10. Standing Council on Health of the Australian Health Ministers' Conference. National Strategic Framework for Rural and Remote Health. Canberra: Commonwealth of Australia, 2012.

11. Middleton S, Gardner A, Gardner G, et al. The status of Australian nurse practitioners: the second national census. Aust Health Rev 2011;35:448.

12. Barnason S, Morris K. Health care in rural hospitals: a role for nurse practitioners. Adv Emerg Nurs J 2011;33:145-54.

13. Gardner G, Gardner A, O'Connell J. Using the Donabedian framework to examine the quality and safety of nursing service innovation. J Clin Nurs 2014;23:145.

14. Donabedian A. Evaluating the quality of medical care. Milbank Mem Fund Q 1966;44(Suppl):166-206.

15. Donabedian A. The quality of care: how can it be assessed? JAMA 1988;260:1743-8.

16. Lawson EF, Yazdany J. Healthcare quality in systemic lupus erythematosus: using Donabedian conceptual framework to understand what we know. Int J Clin Rheumtol 2012;7:95-107.

17. Hood MN. A review of cohort study design for cardiovascular nursing research. J Cardiovasc Nurs 2009;24:E1-9.

18. Silverman SL. From randomized controlled trials to observational studies. Am J Med 2009;122:114-20.

19. Horn SD, Gassaway J. Practice-based evidence study design for comparative effectiveness research. Med Care 2007;45(10 Supl 2): S50-7.

20. Soh SE, Saw SM. Cohort studies: design and pitfalls. Am J Ophthalmol 2010;150:3-5.

21. World Health Organisation. International Statistical Classification of Diseases and Related Health Problems 10th Revision (ICD-10) 
Version for 2010-Chapter IX Diseases of the Circulatory System 2010 (cited 9 October 2014). http://apps.who.int/classifications/ icd10/browse/2010/en\#/I20-I25

22. Gardner G, Gardner A, Middleton S, et al. AUSPRAC: The Nurse Practitioner Research Toolkit, Australian College of Nurse Practitioners. 2010. www.acnp.org.au/images/stories/pdfs/ausprac_ np_toolkit.pdf

23. Quality Metric. SF-12v2 Health Survey (9 October 2014). http://www. qualitymetric.com/WhatWeDo/SFHealthSurveys/SF12v2Health Survey/tabid/186/Default.aspx

24. Snellman I, Jonsson B, Wikblad K. Validation and test-retest reliability of a health measure, health as ability of acting, based on the welfare theory of health. Eval Health Prof 2012;35:87-103.

25. Ware JJ, Kosinski M, Keller SD. A 12-Item Short-Form Health Survey: construction of scales and preliminary tests of reliability and validity. Med Care 1996;34:220-33.
26. Fisher JD. Use of the 12-Item Short-Form (SF-12) Health Survey in an Australian Heart and Stroke population. Qual Life Res 1999;8:1-8.

27. Sanderson K, Andrews G. The SF-12 in the Australian population: cross-validation of item selection. Aust N Z J Public Health 2002;26:343-5.

28. Cheung TK, Leung GY, Yuen MF, et al. Quality of life and psychological impact in patients with noncardiac chest pain. J Clin Gastroenterol 2009;43:13-18.

29. Dinh M, Walker A, Parameswaran A, et al. Evaluating the quality of care delivered by an emergency department fast track unit with both nurse practitioners and doctors. Australas Emerg Nurs $J$ 2012:15:188.

30. Roche T, Gardner G, Lewis PA. A retrospective observational study of patients who present to Australian rural emergency departments with undifferentiated chest pain. Aust J Rural Health 2014;22:229-34. 